\title{
Propagation properties of Rossby waves for latitudinal $\beta$-plane variations of $f$ and zonal variations of the shallow water speed
}

\author{
C. T. Duba ${ }^{1}$ and J. F. McKenzie ${ }^{1,2}$ \\ ${ }^{1}$ Department of Mathematics, Statistics and Physics, Durban University of Technology, P.O. Box 1134, Durban, South Africa \\ ${ }^{2}$ School of Mathematical Sciences, University of Kwazulu Natal, Private Bag X54001, Durban, 4000, South Africa
}

Correspondence to: C. T. Duba (thamadub@dut.ac.za)

Received: 4 December 2011 - Revised: 7 April 2012 - Accepted: 17 April 2012 - Published: 15 May 2012

\begin{abstract}
Using the shallow water equations for a rotating layer of fluid, the wave and dispersion equations for Rossby waves are developed for the cases of both the standard $\beta$ plane approximation for the latitudinal variation of the Coriolis parameter $f$ and a zonal variation of the shallow water speed. It is well known that the wave normal diagram for the standard (mid-latitude) Rossby wave on a $\beta$-plane is a circle in wave number $\left(k_{\mathrm{y}}, k_{\mathrm{x}}\right)$ space, whose centre is displaced $-\beta / 2 \omega$ units along the negative $k_{\mathrm{x}}$ axis, and whose radius is less than this displacement, which means that phase propagation is entirely westward. This form of anisotropy (arising from the latitudinal $y$ variation of $f$ ), combined with the highly dispersive nature of the wave, gives rise to a group velocity diagram which permits eastward as well as westward propagation. It is shown that the group velocity diagram is an ellipse, whose centre is displaced westward, and whose major and minor axes give the maximum westward, eastward and northward (southward) group speeds as functions of the frequency and a parameter $m$ which measures the ratio of the low frequency-long wavelength Rossby wave speed to the shallow water speed. We believe these properties of group velocity diagram have not been elucidated in this way before. We present a similar derivation of the wave normal diagram and its associated group velocity curve for the case of a zonal $(x)$ variation of the shallow water speed, which may arise when the depth of an ocean varies zonally from a continental shelf.
\end{abstract}

Keywords. Electromagnetics (Wave propagation)

\section{Introduction}

The propagation properties of mid-latitude Rossby waves on a $\beta$-plane are well known (Gill, 1982; Pedlosky, 1987). The dispersion equation in either its diagnostic form, $(\omega, k)$ plots, or wave normal form (Longuet-Higgins, 1964) shows that phase propagation is purely westward and that the waves cannot propagate above a critical frequency, at which the zonal group velocity becomes zero for a wave number equal to the inverse Rossby radius. This implies that for wavelengths less (greater) than the Rossby radius, the zonal group velocity is eastward (westward), while the phase velocity remains westward. This "backward" property, i.e. phase and group velocities in opposite directions, also manifests itself at a general angle of phase propagation, in that poleward directed rays (energy flux direction) correspond to equatorward wave normal (or phase) directions.

In this paper we highlight these anisotropic and dispersive properties through the use of the phase and group velocity diagrams, in which the former takes the well known form of a circle, whose centre is displaced westward and the latter, the less well known form, is an ellipse, whose centre is also displaced westward. The important parameters are the wave frequency $\omega$ (suitably normalized) and a parameter $m$, which is the ratio of the Rossby wave zonal speed (at low frequencies and long wavelengths) to the shallow water speed.

In the next section we use the shallow water equations to derive the wave equation for the system, which reduces to the classical Rossby wave equation in the low frequency approximation. This leads to the well-known dispersion equation at mid-latitudes and is generalized to include not only the usual $\beta$-effect arising from the latitudinal variation of the 
Coriolis force, but also a topographic $\beta$-effect arising from background variations in the shallow water speed.

In Sect. 3 we outline the propagation properties of the midlatitude Rossby wave in terms not only of its well-known wave normal diagram (Longuet-Higgins, 1964), but also its phase and group velocity diagrams. Of particular interest is the latter, which is normally given as expressions for the zonal and latitudinal group speeds in terms of the wave number vectors, which can be reduced to the zonal wave normal as the generating parameter. At a given frequency we show that the group velocity, $\left(V_{\mathrm{gy}}, V_{\mathrm{gx}}\right)$, curve is in fact an ellipse, with centre displaced westward by an amount that depends on the " $m$ " of the planet and the ratio of the wave frequency to the critical frequency, above which Rossby waves are evanescent. The major and minor axes yield the maximum zonal, westward, eastward and northward speeds as functions of frequency and $m$. The corresponding phase velocity diagram is a circle, with centre displaced westward. In the limiting case of $m \rightarrow \infty$ (or infinite Rossby radius), the group velocity curve becomes a parabola, and the phase velocity curve becomes a line indicating constant zonal phase velocity for all directions of propagation (limited of course to the 2 nd and 3rd quadrants). These results are supplemented by the relation between the ray direction $\chi$ and the wave normal angle $\phi$. It is well known that the latitudinal components of the phase and group velocities are anti-parallel. Interestingly, this "backward" property of the Rossby wave has been invoked to explain the dipole-like formation of equatorial easterly jets resulting from localized equatorial heating and at higher latitudes westerly jets (Diamond et al., 2008).

In Sect. 4 we carry out similar calculations for a topographic $\beta$-effect arising from zonal variations of the shallow water speed. In this case, it is as if the properties described in Sect. 3 were rotated through $\pi / 2$ from west to north.

\section{The general Poincaré-Rossby wave equation and the low frequency Rossby wave}

The linearised shallow water equations in a rotating layer of fluid of depth $H(x, y)$ may be written (Gill, 1982; Pedlosky, 1987)

$$
\frac{\partial \underline{Q}}{\partial t}+\underline{f} \times \underline{Q}=-c^{2} \nabla \eta
$$

$$
\frac{\partial \eta}{\partial t}+\operatorname{div} \underline{Q}=0
$$

in which $Q=\left(Q_{\mathrm{x}}, Q_{\mathrm{y}}\right)=H(u, v)$ is the perturbation horizontal momentum vector, $(u, v)$ the horizontal velocity, $\eta$ the displacement of the surface from its equilibrium depth $H, f=2 \Omega(\sin \theta) \hat{z}$ is the Coriolis parameter $(\Omega$ rotation frequency) and $c=\sqrt{g H}$ the shallow water speed. The operations $\frac{\partial}{\partial t} \operatorname{div}$ on Eq. (1) plus $f$ times the $z$ component of the curl of Eq. (1), and the use of Eq. (2) to eliminate $\operatorname{div} Q$ in favour of $-\partial \eta / \partial t$, and Eq. (1) to eliminate $Q$ in terms of $\nabla \eta$ immediately lead to the following wave equation for the displacement $\eta$ :

$$
\frac{\partial}{\partial t}\left[\left(\frac{\partial^{2}}{\partial t^{2}}+f^{2}\right) \eta-\operatorname{div}\left(c^{2} \nabla \eta\right)\right]=\left(\nabla\left(c^{2} f\right) \times \nabla \eta\right)_{\mathrm{z}},
$$

in which $f$ and $c$ may be functions of $x$ and $y$. Equation (3) is the general equation for the combined system of PoincaréRossby waves (Pedlosky, 1987). In the "low-frequency" approximation $\left(\frac{\partial}{\partial t}<<f\right)$, this equation reduces to the classical Rossby wave equation:

$$
\frac{\partial}{\partial t}\left[\frac{f^{2}}{c^{2}}-\left(\frac{\partial^{2}}{\partial x^{2}}+\frac{\partial^{2}}{\partial y^{2}}\right)\right] \eta=(\underline{\beta} \times \nabla \eta)_{\mathrm{z}},
$$

in which

$$
\underline{\beta} \equiv\left(\beta_{\mathrm{x}}, \beta_{\mathrm{y}}\right)=c^{-2} \nabla\left(c^{2} f\right)
$$

since $\nabla f=(0, \beta, 0)$. Equation (4) is a generalization of the usual Rossby wave equation (see Pedlosky, 1987) to include the topographic " $\beta$ " effect through spatial variations (in $x$ and $y$ ) of the shallow water speed $c$ as well as the $y$ variation of $f\left(=f_{0}+\beta y\right)$ in the classical $\beta$-plane approximation of the latitudinal variation of the vertical component $f \hat{z}$ on a spherical planet of radius $R$ where

$f=f_{0}+\beta_{0} y, \quad \beta_{0}=\frac{2 \Omega \cos \theta_{0}}{R}, \quad f_{0}=2 \Omega \sin \theta_{0}$.

Here $\theta_{0}$ is the latitude at which the $\beta$-plane is constructed tangent to the surface at $\theta_{0}$, and therefore $y$ measures distance northward whilst $x$ is directed eastward.

If the background variations of $c^{2}(x, y)$ and $f(y)$ are assumed "slow" over a wavelength, the wave Eq. (4) admits a local dispersion relation for plane waves, varying as $\exp i\left(\omega t-k_{x} x-k_{y} y\right)$ namely

$$
\omega=\frac{-\beta_{\mathrm{y}} k_{\mathrm{x}}+\beta_{\mathrm{x}} k_{\mathrm{y}}}{\left(k_{\mathrm{x}}^{2}+k_{\mathrm{y}}^{2}+f_{0}^{2} / c^{2}\right)}
$$

or

$\left(k_{\mathrm{y}}-\frac{\beta_{\mathrm{x}}}{2 \omega}\right)^{2}+\left(k_{\mathrm{x}}+\frac{\beta_{\mathrm{y}}}{2 \omega}\right)^{2}=\frac{\beta_{\mathrm{x}}^{2}+\beta_{\mathrm{y}}^{2}}{4 \omega^{2}}-\frac{f_{0}^{2}}{c^{2}}$.

For a given $\omega$ the latter wave normal form is a circle centred at $\left(-\beta_{\mathrm{y}} / 2 \omega, \beta_{\mathrm{x}} / 2 \omega\right)$ of radius given by the square root of the RHS of Eq. (8). The presence of an inhomogeneity in $x$ (zonal eastward), $d c^{2} / d x$, gives rise to a displacement of the usual Rossby wave, representing purely westward propagation $\left(k_{\mathrm{x}}<0\right)$, northward so as to permit eastward phase 


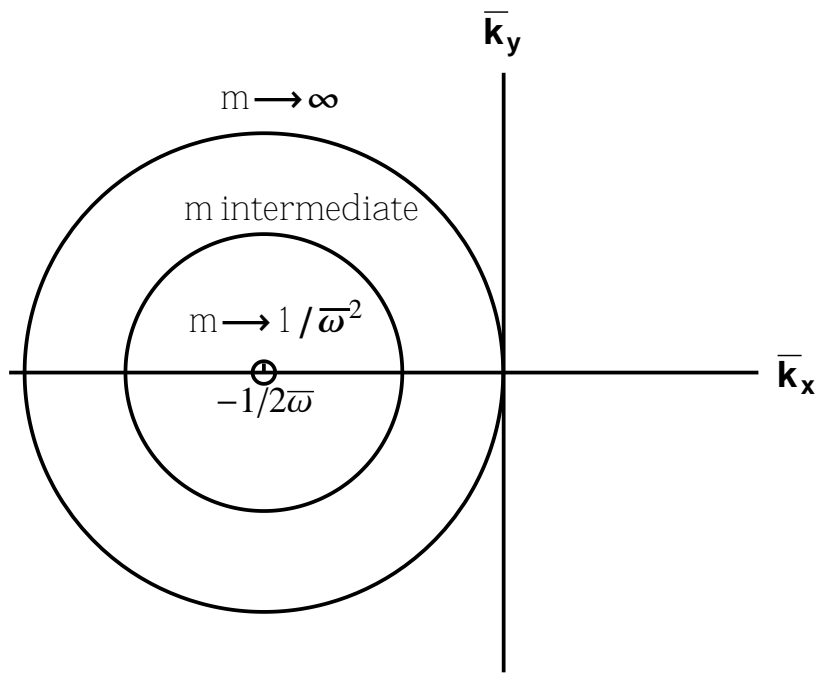

Fig. 1. The wave normal circle for various values of $m$.

propagation $\left(k_{\mathrm{x}}>0\right)$, as represented by any $\underline{k}$ pointing northeast with a corresponding ray directed southwest. Thus, a northwest propagation vector $\underline{k}$ gives rise to a ray directed southwest. Such are the rather peculiar propagation properties of the Rossby wave, which follow from the theorem that the group velocity vector points along the normal to the wave normal curve in the direction of increasing $\omega$ (Lighthill, 1978). We shall now explore these properties in greater detail through the use of figures representing the wave normal diagram and the phase and group velocity diagrams at given frequencies for different values of $m$.

\section{Propagation properties of the mid-latitude Rossby wave: wave normal, phase and group velocity curves for $\beta_{\mathbf{y}} \neq 0\left(\beta_{\mathrm{x}}=\mathbf{0}\right)$}

The propagation properties of the Rossby wave have been discussed extensively, for example, in the texts by Gill (1982) and Pedlosky (1987). Here we develop the group velocity diagram at a fixed $\omega$, which provides the counterpart to the wave normal diagram. In the case where the Rossby wave arises from inhomogeneity only in the y-direction $\left(\beta_{\mathrm{x}}=0\right)$, the diagnostic $(\bar{\omega}, \bar{k})$ plot and the wave normal curves are given by

$\bar{\omega}=\frac{-\bar{k}_{\mathrm{x}}}{\bar{k}^{2}+1 / m}$,

and Eq. (8) may be written

$\bar{k}_{\mathrm{y}}^{2}+\left(\bar{k}_{\mathrm{x}}+\frac{1}{2 \bar{\omega}}\right)^{2}=\frac{1}{4 \bar{\omega}^{2}}-\frac{1}{m}$,

in which $\bar{\omega}$ is the normalized frequency, $\omega / \sqrt{\beta_{\mathrm{y}} c}, \quad \overline{\underline{k}}$, the normalized wave number vector $\underline{k}\left(c / \beta_{\mathrm{y}}\right)^{1 / 2}$ and $m$ is given by

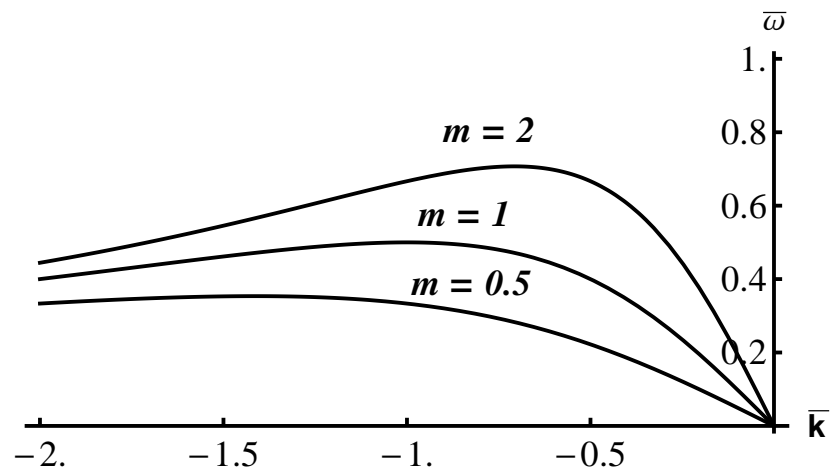

Fig. 2. The diagnostic diagram $(\bar{\omega}, \bar{k})$ plot for various values of $m$.

$m \equiv \beta_{\mathrm{y}} c / f_{0}^{2}=\frac{\cos \theta_{0}}{\sin ^{2} \theta_{0}} \frac{1}{2 M}$,

$M=\Omega R / c$.

Here we have assumed that there is no latitudinal variation of $c^{2}$, and therefore the $\beta$ effect arises solely from the variation of $f$ as in Eq. (6); $M$ is a Mach (or Froude) number measuring the equatorial rotation speed $\Omega R$ in units of the shallow water speed $c$, and $m$ is in fact the ratio of the Rossby zonal phase speed (at low frequencies and long wavelengths) to $c$. Equation (10) is a circle in $\left(\bar{k}_{\mathrm{y}}, \bar{k}_{\mathrm{x}}\right)$ space of radius $\sqrt{1 / 4 \bar{\omega}^{2}-1 / m}$, whose centre is displaced along the negative $\bar{k}_{\mathrm{x}}$ axis by $-1 / 2 \bar{\omega}$ units (Longuet-Higgins, 1964), as shown in Fig. 1 along with the $(\bar{\omega}, \bar{k})$ plot in Fig. 2. Note that the phase propagation is entirely westward and propagation requires $\bar{\omega}<\sqrt{m} / 2$. Figure 3 demonstrates the geometrical relation between the ray direction $\chi$ and the wave normal angle $\phi$, both measured from $\bar{k}_{\mathrm{x}}$-axis, which shows the two values of $\chi$ for any given $\phi$. The relationship between $\chi$ and $\phi$ is found by expressing $\chi$ as normal to the slope of the $\left(\bar{k}_{\mathrm{y}}, \bar{k}_{x}\right)$ curve, i.e. $\tan \chi=-1 /\left(\partial \bar{k}_{\mathrm{y}} / \partial \bar{k}_{\mathrm{x}}\right)$, which yields

$\tan \chi=(\sin \phi \cos \phi) /\left(\cos ^{2} \phi+\frac{1}{-1 \pm \sqrt{1-4 \omega^{2} / m \cos ^{2} \phi}}\right)$.

For a given $\phi$ there are two values of $\chi$, as shown in Fig. 4 . We also show the limiting case $m \rightarrow \infty$, which yields $\chi=$ $2 \phi$ (lower sign $m$ in Eq. 13) and $\chi=\pi$ (upper sign). In this limit $f_{0}{ }^{2} / c^{2} \rightarrow 0$, and the Rossby wave Eq. (4) reduces to the classic form (see e.g. Yagamata, 1976)

$\frac{\partial}{\partial t} \nabla^{2} \psi=-\beta \frac{\partial \psi}{\partial x}$

where $\psi$ is the stream function corresponding to strict 2-D compressibility. It is for this reason we include a discussion in the limit $m \rightarrow \infty$. 


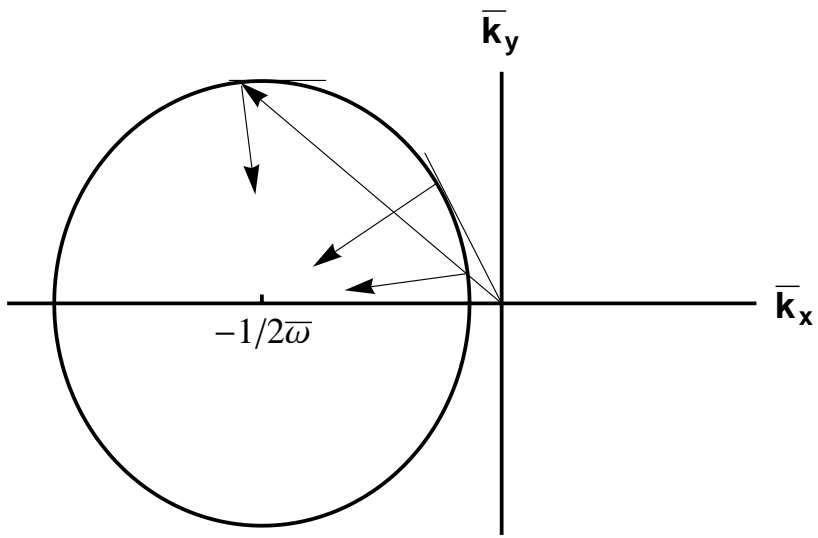

Fig. 3. The wave normal displaced circle showing the relation between the ray direction $\chi$ (given by the arrows pointing towards the centre measured from $\bar{k}_{\mathrm{X}}$ ) and the wave normal angle $\phi$ between $\underline{k}$ and $\bar{k}_{\mathrm{X}}$.

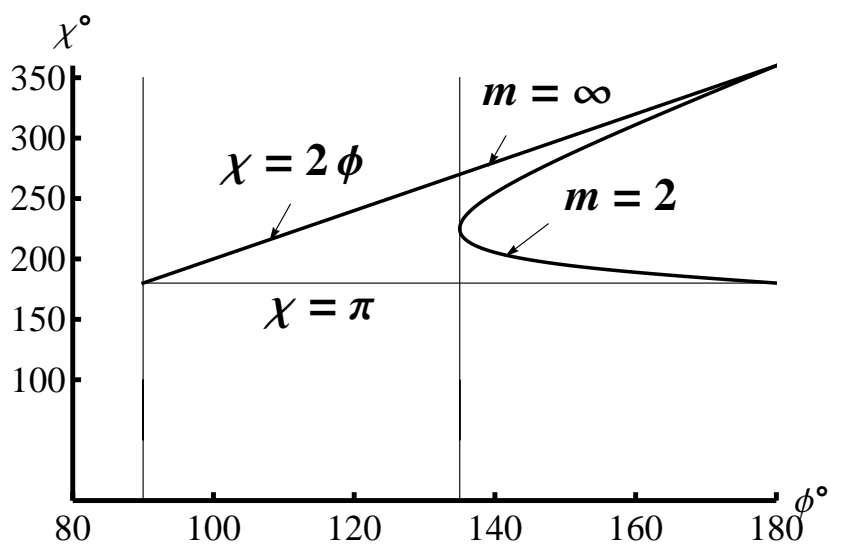

Fig. 4. The variation of $\chi$ (two values) with $\phi$ for $m=2$.

The phase velocity $V_{\mathrm{p}}=\omega / k$, which follows immediately from Eq. (7), may be written in the normalized form

$\bar{V}_{\mathrm{py}}^{2}+\left(\bar{V}_{\mathrm{px}}+\frac{m}{2}\right)^{2}=\frac{m^{2}}{4}\left(1-\frac{4 \bar{\omega}^{2}}{m}\right)$,

in which $V_{\mathrm{p}}$ has been normalized with respect to the shallow water speed $c$. Thus, the phase velocity diagram is a circle of radius $\frac{m}{2} \sqrt{1-4 \bar{\omega}^{2} / m}$, whose origin is displaced westward by $-m / 2$ units and therefore lies entirely in the regime of westward propagation. The smallest value of the westward $\bar{V}_{\text {px }}$ (in which $\bar{V}_{\text {py }}=0$ in Eq. 15) is

$\bar{V}_{\mathrm{pxmin}}=\frac{-m}{2}+\frac{m}{2} \sqrt{1-\frac{4 \bar{\omega}^{2}}{m}}$,

which approaches $-\bar{\omega}^{2}$ as $m \rightarrow \infty$. Thus, in the limit $m \rightarrow$ $\infty$ (which corresponds to infinite Rossby radius or Rossby wave speed greatly in excess of the shallow water speed)

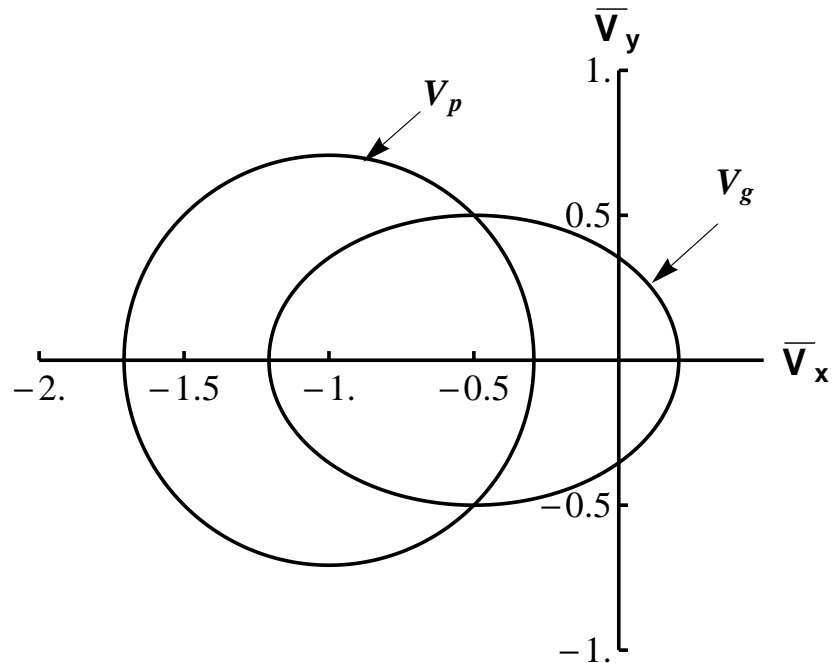

Fig. 5. The phase velocity $V_{\mathrm{p}}$ (a circle) and group velocity $V_{\mathrm{g}}$ (an ellipse) diagrams for a fixed $\bar{\omega}$ and $m=2$.

the phase velocity diagram reduces to the line $\bar{V}_{\mathrm{px}}=-\bar{\omega}^{2}$ in $\left(\bar{V}_{\mathrm{py}}, \bar{V}_{\mathrm{px}}\right)$ space.

The group velocity $\underline{V}_{\mathrm{g}}=\partial \omega / \partial \underline{k}$ follows from Eq. (7) (in which we put $\beta_{\mathrm{x}}=0$ ) in the form

$$
\begin{aligned}
& V_{\mathrm{gx}}=\frac{\beta\left(k_{\mathrm{x}}{ }^{2}-\left(k_{\mathrm{y}}{ }^{2}+f_{0}^{2} / c^{2}\right)\right)}{\left(k_{\mathrm{x}}^{2}+k_{\mathrm{y}}{ }^{2}+f_{0}^{2} / c^{2}\right)^{2}}, \\
& V_{\mathrm{gy}}=\frac{2 \beta k_{\mathrm{y}} k_{\mathrm{x}}}{\left(k_{\mathrm{x}}^{2}+k_{\mathrm{y}}{ }^{2}+f_{0}^{2} / c^{2}\right)^{2}} .
\end{aligned}
$$

In the classic texts (Pedlosky, 1987; Gill, 1972) the group velocity is left in this less than perspicacious parametric form, in which the $\bar{k}_{\mathrm{x}}$ is the generating parameter, with $\bar{k}_{\mathrm{y}}$ given in terms of $\bar{k}_{\mathrm{x}}$ from the dispersion equation. Here we show that the group velocity diagram is in fact an ellipse. This simple result follows from a few algebraic steps by eliminating the denominator from Eqs. (17) and (18) using the dispersion equation, so that in normalized form, Eqs. (17) and (18) become

$\bar{V}_{\mathrm{gx}}=\bar{\omega}^{2}\left(2+1 / \bar{\omega} k_{\mathrm{x}}\right)$,

$\bar{V}_{\mathrm{gy}}=2 \bar{\omega}^{2}\left(\bar{k}_{\mathrm{y}} / \bar{k}_{\mathrm{x}}\right)$.

It is now straightforward to eliminate $\bar{k}_{\mathrm{x}}$ in favour of $\bar{V}_{\mathrm{gx}}$ from Eq. (19), which on substitution into the square of Eq. (20) gives directly the group velocity $\left(\bar{V}_{\mathrm{gx}}, \bar{V}_{\mathrm{gy}}\right)$ curve in the form

$\bar{V}_{\mathrm{gy}}^{2}=4 \bar{\omega}^{4}\left[\left(1-\frac{\bar{V}_{\mathrm{gx}}}{\bar{\omega}^{2}}\right)-\frac{\bar{\omega}^{2}}{m}\left(\frac{\bar{V}_{\mathrm{gx}}}{\bar{\omega}^{2}}-2\right)^{2}\right]$, 


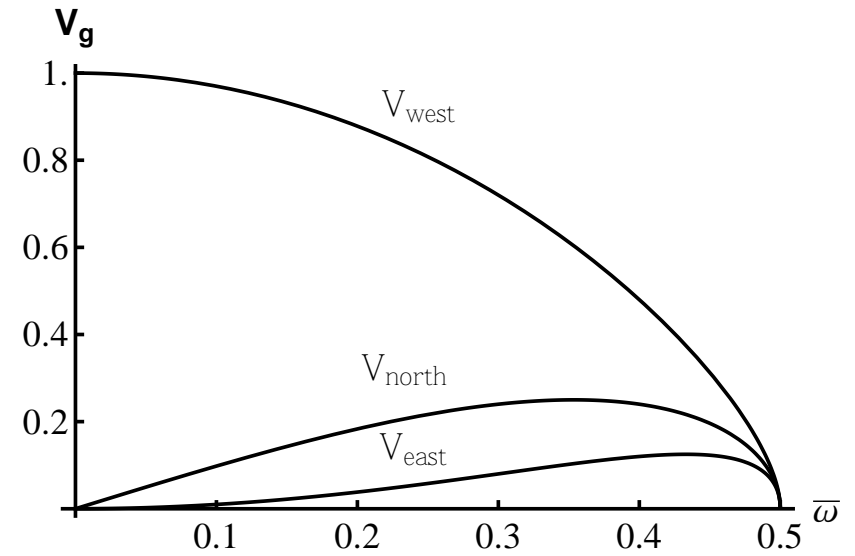

Fig. 6. The maximum westward, northward and eastward group speeds as a function of frequency $\bar{\omega}$ for $m=1$.

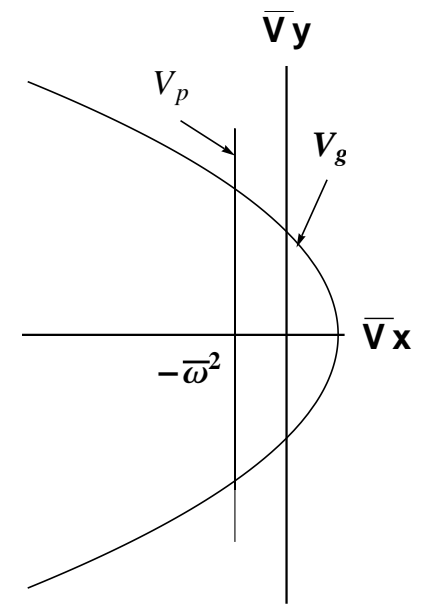

Fig. 7. The group velocity (a parabola) and phase velocity (a line) diagrams for the case $m \rightarrow \infty$.

which may also be written as

$\frac{m \bar{V}_{\mathrm{gy}}^{2}}{4 \bar{\omega}^{2}}+\left[\bar{V}_{\mathrm{gx}}+\frac{m}{2}\left(1-\frac{4 \bar{\omega}^{2}}{m}\right)\right]^{2}=\frac{m^{2}}{4}\left(1-\frac{4 \bar{\omega}^{2}}{m}\right)$.

The group velocity curve is an ellipse, whose centre is displaced $\frac{-m}{2}\left(1-4 \bar{\omega}^{2} / m\right)$ units along the $\bar{V}_{\mathrm{gx}}$ axis. The phase and group velocity curves are shown in Fig. 5. The southward group velocity is simply a reflection of the northward group velocity in the $\mathrm{x}$-axis. The maximum eastward and westward group speeds are given by

$\bar{V}_{\mathrm{gx}}^{ \pm}=\frac{m}{2}\left(1-\frac{4 \bar{\omega}^{2}}{m}\right)^{1 / 2}\left[ \pm 1-\left(1-\frac{4 \bar{\omega}^{2}}{m}\right)^{1 / 2}\right]$, and the maximum northward (southward) group speed also follows as

$\bar{V}_{\text {gymax }}= \pm \bar{\omega} m^{1 / 2}\left(1-\frac{4 \bar{\omega}^{2}}{m}\right)^{1 / 2}$.

Examples of the behaviour of extremal group velocities $\bar{V}_{\text {pxmin }}, \bar{V}_{\text {pxmax }}$ and $\bar{V}_{\text {pymax }}$ are shown in Fig. 6. Note that the ellipse collapses to the origin as $m \rightarrow 4 \bar{\omega}^{2}$, at which the wave normal collapses to the point $k_{\mathrm{x}}=-1 / 2 \bar{\omega}$. In the limiting case in which $m>>1$, which can prevail quite near the equator $\left(\theta_{0} \rightarrow 0\right)$, Eq. (21) tends to the parabola

$\bar{V}_{\mathrm{gy}}= \pm 2 \bar{\omega}^{2}\left(1-\frac{\bar{V}_{\mathrm{gx}}}{\bar{\omega}^{2}}\right)^{1 / 2}$,

which is shown in Fig. 7 together with the phase velocity diagram, which is simply the line $V_{\mathrm{px}}=-\bar{\omega}^{2}$. It is of some interest to emphasize that Rossby waves are "backward" in the sense that the latitudinal components of their phase and group velocities are always in opposite directions. This property can be invoked to describe the formation of a dipole pair of jets in the following way: Northward (away from the equator) wave energy flux is associated with southward, towards the equator, wave momentum flux; and the opposite in the case of southward directed energy flux, away from the equator, corresponds to northward (towards the equator) momentum flux. In other words, a poleward energy flux from the equator is associated with an equatorward flux of momentum. Hence, Rossby wave dynamics implies that localized equatorial heating gives rise to equatorial easterly zonal jets. This "convergence" of equatorial momentum implies a deficit at higher latitudes such that a westerly jet must necessarily form there (Diamond et al., 2008).

\section{Propagation properties of the topographic Rossby wave: wave normal, phase and group velocity curves for $\beta_{\mathrm{x}} \neq \mathbf{0}\left(\beta_{\mathrm{y}}=\mathbf{0}\right)$}

In this case a zonal inhomogeneity ( $c$ a function of $x$ ) dispersion and wave normal curves given by putting $\beta_{\mathrm{y}}=0$ in Eqs. (7) and (8), are

$\bar{\omega}=\frac{\bar{k}_{\mathrm{y}}}{\bar{k}_{\mathrm{x}}^{2}+\bar{k}_{\mathrm{y}}^{2}+1 / m}$,

$\bar{k}_{\mathrm{x}}^{2}+\left(\bar{k}_{\mathrm{y}}-\frac{1}{2 \bar{\omega}}\right)^{2}=\frac{1}{4 \bar{\omega}^{2}}-\frac{1}{m}$,

in which $\omega$ and $k$ have been normalized to $\sqrt{\beta_{\mathrm{X}} c}$ and $\left(c / \beta_{\mathrm{X}}\right)^{1 / 2}$, respectively and $m$ is now given by

$m=\beta_{\mathrm{x}} \frac{c}{f_{0}^{2}}$, 


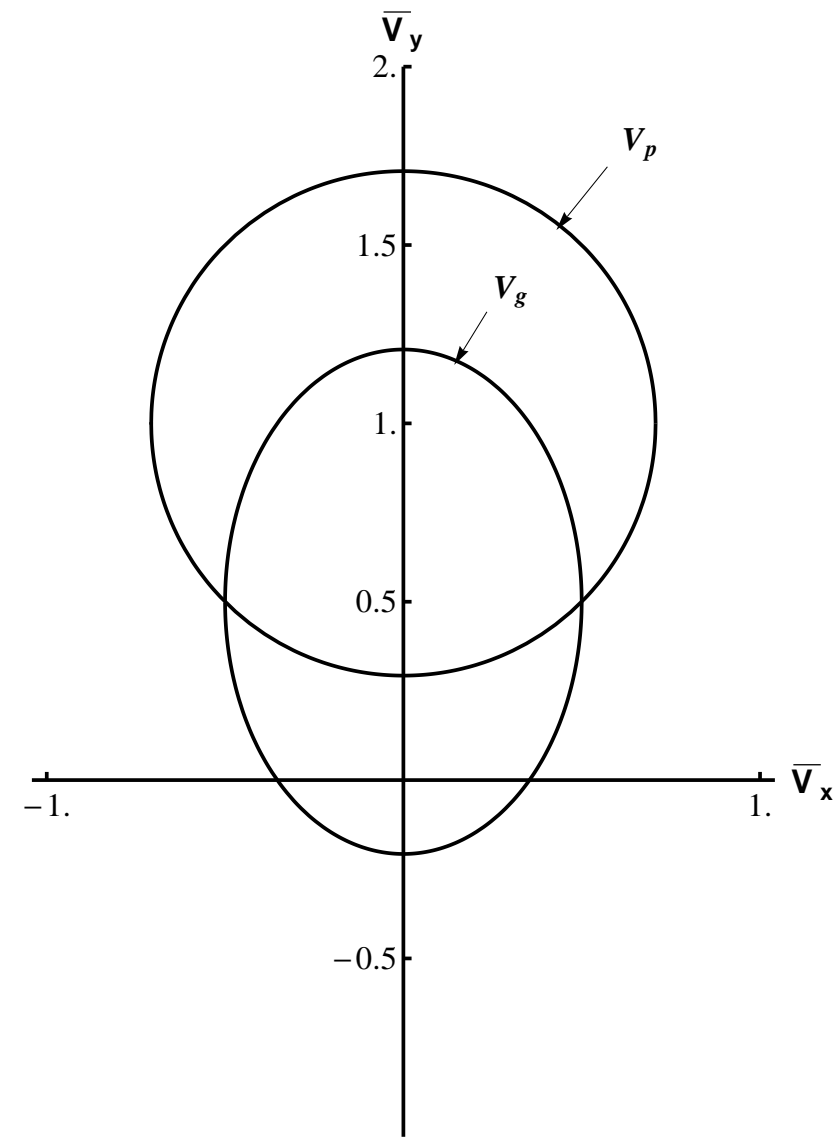

Fig. 8. The phase and group velocity diagrams for topographic Rossby waves where shallow water speed varies zonally for fixed $\bar{\omega}$ and $m=2$.

$\beta_{\mathrm{x}}=\frac{f_{0}}{c^{2}} \frac{d c^{2}}{d x}$.

The wave normal diagram is again a circle, but now its centre is displaced $1 / 2 \bar{\omega}$ units along the positive $\bar{k}_{\mathrm{y}}$ axis. The corresponding phase velocity diagram is given by

$$
\left(\bar{V}_{\mathrm{py}}-\frac{m}{2}\right)^{2}+\bar{V}_{\mathrm{px}}^{2}=\frac{m^{2}}{4}\left(1-\frac{4 \bar{\omega}^{2}}{m}\right),
$$

which is a circle of radius $\frac{m}{2}\left(1-4 \bar{\omega}^{2} / m\right)^{1 / 2}$ with centre displaced $m / 2$ units along the $\bar{V}_{p y}$ axis. Hence, phase propagation is always northward, although as we can now anticipate, there are both northward and southward components of the group velocity. It is as if the classical westward Rossby wave phase were rotated through ninety degrees. The calculation of the group velocity follows similar lines but now with

$\bar{V}_{\mathrm{gy}}=\bar{\omega}^{2}\left(-2+\frac{1}{\bar{\omega} \bar{k}_{\mathrm{y}}}\right)$,
$\bar{V}_{\mathrm{gx}}=-2 \bar{\omega}^{2} \bar{k}_{\mathrm{x}} / \bar{k}_{\mathrm{y}}$

Hence, the group velocity curve is now given by the ellipse

$$
\bar{V}_{\mathrm{gx}}^{2}=4 \bar{\omega}^{4}\left[\left(\frac{\bar{V}_{\mathrm{gy}}}{\bar{\omega}^{2}}+1\right)-\frac{\bar{\omega}^{2}}{m}\left(\frac{\bar{V}_{\mathrm{gy}}}{\bar{\omega}^{2}}+2\right)^{2}\right] \text {, }
$$

or

$$
\frac{m \bar{V}_{\mathrm{gx}}^{2}}{4 \bar{\omega}^{2}}+\left[\bar{V}_{\mathrm{gy}}-\frac{m}{2}\left(1-\frac{4 \bar{\omega}^{2}}{m}\right)\right]^{2}=\frac{m^{2}}{4}\left(1-\frac{4 \bar{\omega}^{2}}{m}\right),
$$

which is shown in Fig. 8. This curve can be obtained by rotating the curve given by Eq. (22) and Fig. 5 through ninety degrees. Thus, the maximum northward, southward and zonal (east or west) speeds are given by Eqs. (23) and (24) in which the labels $x$ and $y$ are interchanged.

\section{Summary}

The propagation properties of mid-latitude Rossby waves are illustrated through the local dispersion equation either in its diagnostic form $((\omega, k)$ plot $)$ or as a wave normal curve for given values of $\omega$, both of which descriptions highlight the dispersive and anisotropic nature of the wave. Here we develop this further by showing that the group velocity diagram is an ellipse, whose centre is displaced westward (Fig. 5) and whose major and minor axis yield the maximum westward, eastward and northward (southward) group speeds as a function of frequency and the parameter $m$ (Fig. 6). This elegant construction replaces the earlier (somewhat cumbersome) expressions for the group velocity, which are usually given in terms of the zonal and northward wave numbers acting as generating parameters as in Eqs. (17) and (18). Similar diagrams exist for the case of topographic Rossby waves, in which shallow water speed varies zonally (Fig. 8). We emphasize that the "backward" property of the Rossby wave has been invoked to explain dipole-like formation of equatorial easterly jets and at higher latitudes westerly jets, as discussed by Diamond et al. (2008).

Acknowledgements. The authors would like to thank the reviewers for the most useful and constructive comments towards the finalization of this paper. This work was supported by the National Research Foundation of South Africa. A thank you note to Clemens Dempers of BlueStallion Technologies who provided technical assistance with the graphics.

Topical Editor C. Jacobi thanks L. Debnath and two other anonymous referees for their help in evaluating this paper.

\section{References}

Diamond, P. H., Gurcan, D. D., Hahn, T. S., Miki, K., and Garbet, $\mathrm{X}$.: Momentum theorems and the structure of atmospheric jets 
and zonal flows in plasmas, Plasma Phys. Control. Fusion, 50, 124018, doi:10.1088/0741-3335/50/12/124018, 2008.

Gill, A. E.: Atmosphere and Ocean Dynamics, Academic Press, London, 662 pp., 1982.

Lighthill, J.: Waves in Fluids, Cambridge University Press, 504 pp., 1978.
Longuet-Higgins, M. S.: On Group Velocity and Energy Flux in Planetary Wave Motions, Deep-Sea Research and Oceanographic Abstracts, 11, pp. 35-42, 1964.

Pedlosky, J.: Geophysical Fluid Dynamics, 2nd Edition, SpringerVerlag, New York, 710 pp., 1987.

Yagamata, T.: On trajectories of Rossby Wave-packets Released in a Lateral Shear Flow, J. Oceanogr. Soc. Japan, 32, 162-168, 1976. 